\title{
The Coupling of Gravity to Spin and Electromagnetism*
}

\author{
Felix Finster, Joel Smoller ${ }^{\dagger}$ and Shing-Tung Yau ${ }^{\ddagger}$
}

March 1999

\begin{abstract}
The coupled Einstein-Dirac-Maxwell equations are considered for a static, spherically symmetric system of two fermions in a singlet spinor state. Stable soliton-like solutions are shown to exist, and we discuss the regularizing effect of gravity from a Feynman diagram point of view.
\end{abstract}

There are some interesting effects that result when one couples gravity, as expressed through Einstein's equations, to other fundamental force fields. The most striking example of this is the discovery by Bartnik and McKinnon (BM) [1] of soliton-like solutions for the $S U(2)$ Einstein-Yang/Mills (EYM) equations. This came as a surprise because several results for related systems lead to the conjecture that such solutions cannot exist [2]. For example, neither the vacuum Einstein equations, nor the Yang/Mills equations admit non-trivial static, globally regular solutions. The existence of the BM solutions, however, is due to the coupling of different force fields, whereby the attractive gravitational force is balanced by the repulsive Yang/Mills force. But this balance is quite delicate, so much so, that the EYM solutions are unstable to small perturbations [3].

We consider here a different type of coupling; namely gravity coupled both to quantum mechanical particles with spin (Dirac particles) and to an electromagnetic field. We analyze the resulting Einstein-Dirac-Maxwell (EDM) equations for a static, spherically symmetric system of two fermions in a singlet spinor state. We construct stable soliton-like solutions, and we discuss some of their properties for different values of the electromagnetic coupling constant. Our aim is to show that the gravitational interaction has a certain "regularizing" effect; this will be explained from a Feynman diagram point of view.

Before going further, we point out that throughout this essay, we are considering only classical fields coupled to the Dirac equation; in particular, we consider neither quantized bosonic fields, nor second quantization of the Dirac field.

*This essay received an "honorable mention" in the 1999 Essay Competition of the Gravity Research Foundation - Ed.

${ }^{\dagger}$ Research supported in part by the NSF, Grant No. DMS-G-9802370.

₹esearch supported in part by the NSF, Grant No. 33-585-7510-2-30. 
The EDM equations for a system of $n$ Dirac particles have the following general form,

$$
\begin{aligned}
R_{j}^{i}-\frac{1}{2} R \delta_{j}^{i} & =-8 \pi T_{j}^{i} & & \text { (Einstein equations) } \\
\left(i G^{j} D_{j}-m\right) \Psi_{a} & =0, \quad a=1, \ldots, n & & \text { (Dirac equations) } \\
\nabla_{l} F^{k l} & =4 \pi j^{k} & & \text { (Maxwell equations) }
\end{aligned}
$$

where $j^{k}=e \sum_{a=1}^{n} \overline{\Psi_{1}} G^{k} \Psi_{a}$ is the Dirac current, and $T_{j}^{i}$ is the sum of the energy momentum tensor of the Dirac particles and of the Maxwell stress-energy tensor. Here $R_{j}^{i}$ is the Ricci tensor, $F_{j k}$ is the electromagnetic (em) field tensor, and $\Psi_{a}$ are the 4-component Dirac wave functions describing particles of mass $m$ and charge $e$. The Dirac matrices $G^{j}$ are $(4 \times 4)$ matrices, which are related to the Lorentzian metric via the anti-commutation relations $\frac{1}{2}\left\{G^{j}(x), G^{k}(x)\right\}=g^{j k}(x) \mathbb{1} . D_{j}$ is the spin connection; it is composed of the Christoffel symbols of the metric connection and of the electromagnetic potential (see [4]). The EDM equations only make physical sense if the wave functions $\Psi_{a}$ are properly normalized. For this, we choose a spacelike hypersurface $\mathcal{H}$ with a future-directed normal vector field $\nu$ and demand that

$$
\int_{\mathcal{H}} \overline{\Psi_{a}} G^{j} \Psi_{b} \nu_{j} d \mu=\delta_{a b} \quad, \quad a, b=1, \ldots, n
$$

where $d \mu$ is the invariant measure on $\mathcal{H}$, induced by the Lorentzian metric.

The Dirac and Maxwell equations are linear in the wave functions and in the electromagnetic field, respectively. But the Dirac current and the energy-momentum tensor are quadratic in the $\Psi_{a}$, the stress-energy tensor is quadratic in the electromagnetic field, and the Einstein equations are nonlinear in the metric. Thus the EDM equations form a highly nonlinear system of differential equations, which in this generality is far too complicated to analyze. Therefore we consider a simple ansatz where the EDM equations reduce to a system of ordinary differential equations; namely a static, spherically symmetric system of two Dirac particles in a singlet spinor state. For the wave functions, "static" means that their time dependence is a plane wave $e^{-i \omega t}$. The parameter $\omega>0$ is the energy of the Dirac particles. Using a special rescaling technique [5, 6], we construct numerical solutions of our EDM system.

We now briefly mention some general properties of the solutions. All of our solutions are everywhere regular, asymptotically Minkowskian, and have finite (ADM) mass $\rho$. The Dirac wave functions decay exponentially at infinity, so that the Dirac particles are with a high probability confined to a neighborhood of the origin; this is due to the gravitational attraction. For large $r$, our solutions look similar to a Reissner-Nordström solution with charge $2 e$ and mass $\rho$. Our solutions are parametrized by two physical parameters: the rest mass $m$ of the Dirac particles and the relative strength of the electromagnetic and gravitational coupling $(e / m)^{2}$. For a fixed value of these parameters, we find an infinite, discrete number of solutions which can be regarded as the ground state, the first excited state, etc. In Figure 1, the mass-energy spectrum for the ground state; i.e., the binding energy $m-\omega$ versus $m$, is plotted for different values of $(e / m)^{2}$; these curves are spirals. 


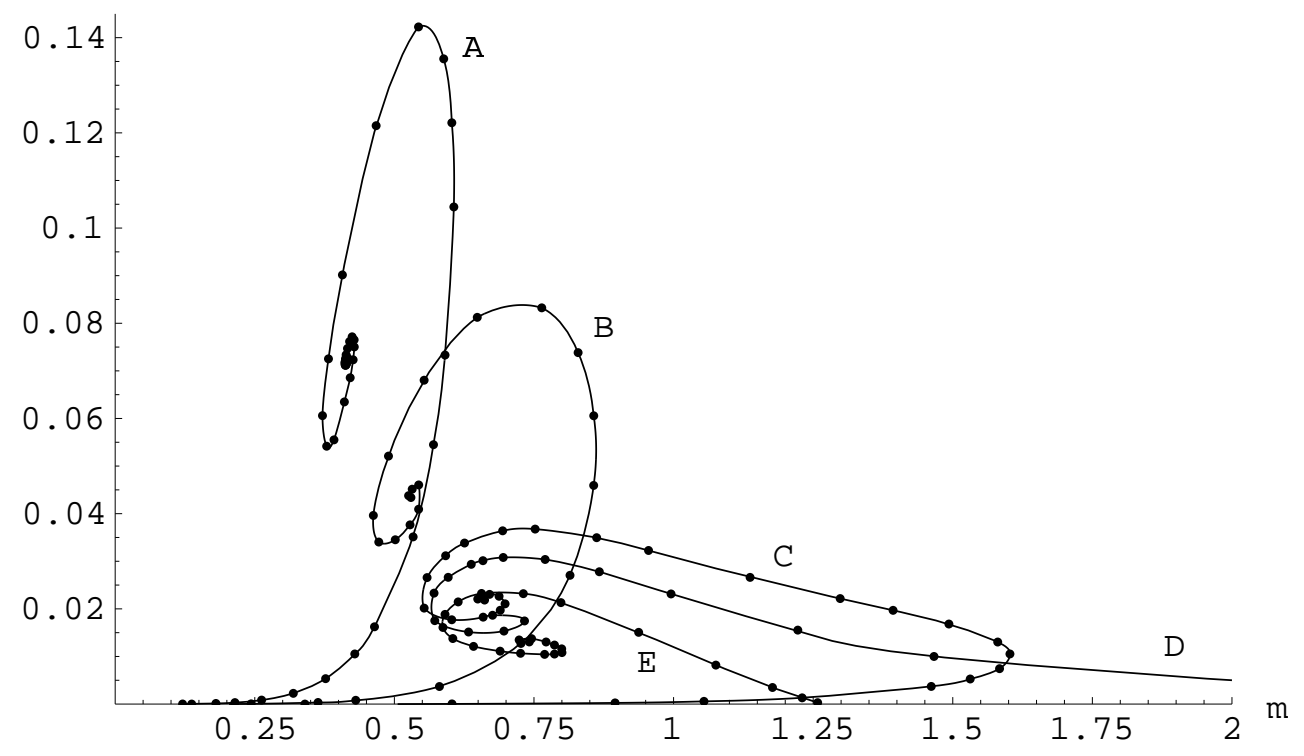

Figure 1: Binding Energy $m-\omega$ of the Fermions for $(e / m)^{2}=0(\mathrm{~A}), 0.7162(\mathrm{~B})$, 0.9748 (C), 1 (D), and $1.0313(\mathrm{E})$.

The binding energy decreases for fixed $m$ as $(e / m)^{2}$ increases; this means that the em repulsion weakens the binding. For $(e / m)>1$, the electromagnetic repulsion is so strong that there are no solutions in the Newtonian limit. However, due to the nonlinearity of Einstein's equations, the EDM equations still admit solutions (spiral E). By combining linear perturbation theory with more subtle topological methods (Conley index, cf. [7]), the stability of the solutions is analyzed [5]. For $(e / m)^{2}<1$, we find that all solutions on the "lower branch" of the spiral (i.e. on the curve from $m=0$ up to the maximal value of $m$ ) are stable, whereas all solutions on the "upper branch" are unstable.

Our solutions of the EDM equations have some interesting features. In this essay, we shall focus on the discussion of what the form of the spiral curves in Figure 1 tells us about the nature of the gravitational interaction (see [5, 6] for the discussion of other effects). If the EDM equations (11) are treated in a perturbation expansion (in the spirit of [8]), we obtain a description of the interaction with Feynman diagrams. More precisely, we get all the Feynman diagrams of quantum field theory, with the only exception of the fermionic loop diagrams; these are absent because we do not consider pair creation/annihilation (the number of Dirac particles is fixed in our ansatz). Thus we can say that, although we consider only classical fields, many aspects of perturbative quantum field theory are already incorporated in our EDM equations. The first question arising from this point of view is why the divergences of the Feynman diagrams do not show up in our regular, well-behaved solutions. To analyze this problem in a simple example, we consider the loop diagram of the virtual emission and absorption of a photon in Figure 2. If evaluated in Minkowski space, this diagram involves the divergent integral over the product of two Green's 


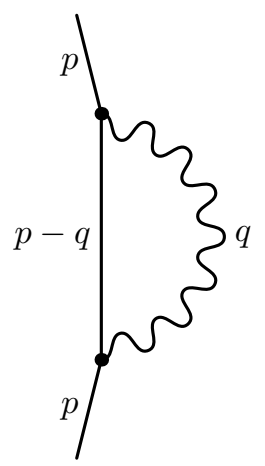

Figure 2: A simple loop diagram

functions,

$$
\lim _{\varepsilon \searrow 0} \int d^{4} q \frac{\left(p^{j}-q^{j}\right) \gamma_{j}+m}{(p-q)^{2}-m^{2}-i \varepsilon p^{0}} \frac{1}{\left(q^{2}-i \varepsilon q^{0}\right)}
$$

In our ansatz, we only consider a static electromagnetic potential, which means that the momentum $q$ of the electromagnetic Green's function must be purely spatial, $q=(0, \vec{q})$. Thus the process is described, instead of (2), by the three-dimensional integral

$$
\lim _{\varepsilon \searrow 0} \int d \vec{q} \frac{p^{0} \gamma_{0}+(\vec{p}-\vec{q}) \vec{\gamma}+m}{\left(p^{0}\right)^{2}-(\vec{p}-\vec{q})^{2}-m^{2}-i \varepsilon p^{0}} \frac{1}{\left(-\vec{q}^{2}-i \varepsilon q^{0}\right)} .
$$

This integral is finite, as one sees by considering the behavior of the integral for large $|\vec{q}|^{2}$, taking into account spherical symmetry. This illustrates that the reason why our interaction is finite is because of our restriction to static, spherically symmetric solutions; note that it is unrelated to the fact that our fields are merely classical.

We now come to our main point: the convergence of the perturbation expansion. A perturbation expansion in the gravitational coupling constant corresponds to a Taylor expansion of our system in the mass parameter $m$. We consider as an example a spiral of Figure 1 for small (fixed) $(e / m)^{2}$. The just explained finiteness of all Feynman diagrams becomes manifest by the fact that the spiral is smooth near the origin. However, since it is not even one-to-one, the spiral is not analytic in $m$. This implies that the perturbation expansion cannot converge absolutely for large values of the coupling constant. This explicit non-convergence result is a peculiar effect of the gravitational interaction; e.g. the mass-energy spectrum for the Dirac-Maxwell system [9] is analytic. Nevertheless, since the spiral curves are bounded, we see that the total interaction is finite even for strong coupling. This implies that there must be cancellations between Feynman diagrams of different order, such that the perturbation series is finite, although this series does not converge absolutely. Thus the gravitational interaction of the Dirac particles is more regular than what one would expect from a naive perturbation expansion. Actually, it has long been conjectured that the ultraviolet divergences of quantum field theory might disappear when gravitation is included in a non-perturbative way (see [10] for a recent formulation of this conjecture). Our EDM system allows us to verify this conjecture explicitly in a simple example. Our results also show that effects like conditional summability 
of the perturbation series actually occur for the fundamental physical interactions with gravity.

\section{References}

[1] Bartnik, R., and McKinnon, J., Phys. Rev. Lett. 61, 141-144 (1988)

[2] Deser, S., Phys. Rev. Lett. B 64, 463 (1976)

[3] Straumann, N. and Zhou, Z., Phys. Lett. B 237, 353-356 (1990)

[4] Finster, F., hep-th/9703083, J. Math. Phys. 39, No. 12, 6276-6290 (1998)

[5] Finster, F., Smoller, J., and Yau, S.-T., gr-qc/9801079 (1998), Phys. Rev. D $59,104020(1999)$

[6] Finster, F., Smoller, J., and Yau, S.-T., gr-qc/9802012 (1998)

[7] Smoller, J., "Shock-Waves and Reaction-Diffusion Equations," 2nd edition, Springer-Verlag, New York (1994)

[8] Bjorken, J. and Drell, S., "Relativistic Quantum Mechanics," McGraw-Hill (1964)

[9] Lisi, G., hep-th/9410244, J. Phys. A 28, 5348 (1995)

[10] Thiemann, T., Class. Quantum Grav. 15, 1281-1314 (1998)

Felix Finster

Max Planck Institute MIS

Inselstr. 22-26

04103 Leipzig, Germany

Felix.Finster@mis.mpg.de
Joel Smoller

Mathematics Department

The University of Michigan

Ann Arbor, MI 48109, USA

smoller@umich.edu
Shing-Tung Yau

Mathematics Department

Harvard University

Cambridge, MA 02138, USA

yau@math.harvard.edu 